\title{
ethic \\ LA RÉCONNAISSANCE COMME IDÉOLOGIE: UNE FAUSSE QUESTION? L'«ANTI-ALTHUSSERIANISME» DE HONNETH ET RANCIÈRE
}

\author{
RECOGNITION AS IDEOLOGY: A WRONG QUESTION? THE \\ "ANTI-ALTHUSSERIANISM" OF HONNETH AND RANCIÈRE
}

\author{
VINICIUS VICENZI' \\ (UFPel/Brasil)
}

\begin{abstract}
RÉSUMÉ
L'article souligne le parallèle entre des formes d'opposition au projet althusserien présentées par Axel Honneth et Jacques Rancière. Cette discussion nous aide à penser un débat Rancière-Honneth capable de comprendre critiquement le concept de reconnaissance aujourd'hui et le concept de sujet qui y est implicite. Nous soulignons l'importance de réfléchir à une reconnaissance non idéologique, dans des conditions de possibilité de subjectivités politiques qui nous garantissent, sous forme de litige, de pouvoir occuper temporairement certains espaces de reconnaissance. Nous cherchons à penser que si cette reconnaissance non idéologique est possible, il faudrait la penser non seulement en termes de justification, d'apprentissage rationnel avec les autres, avec leurs expériences, de place que nous devons occuper comme des êtres de nécessités, de droits semblables et d'estime sociale. L'article est divisé en trois étapes: la première, consacrée à la présentation et à la critique de Honneth à Althusser; la deuxième à la critique de Rancière à la théorie de l'idéologie althusserienne; et la dernière, à la proximité et à l'éloignement entre les deux critiques.
\end{abstract}

Mots clés: Reconnaissance; Idéologie; Sujet; Rancière; Honneth.

\begin{abstract}
The article highlights the parallel between forms of opposition to the Althusserian project presented by Axel Honneth and Jacques Rancière. This discussion helps us to think of a Rancière-Honneth debate capable of critically understanding the concept of recognition today and the concept of subject which is implicit in it. We underline the importance of thinking about a non-ideological recognition, under conditions of possibility of political subjectivities which guarantees, in the form of litigation, manners to temporarily occupy certain spaces of recognition. We seek to think that if this non-ideological recognition is possible, it should be thought of not only in terms of justification, of rational learning with others, with their experiences, of the place that we must occupy as beings of necessity, of similar rights and of social esteem. This article is divided into three stages: the first, devoted to the presentation and criticism of Honneth to Althusser; the second to
\end{abstract}


Rancière's critique of the theory of Althusserian ideology; and the last, to the closeness and distance between the two critics.

Keywords: Recognition; Ideology; Subject; Rancière; Honneth.

\section{Introduction}

Dans son livre La société du mépris (2008), Axel Honneth consacre tout un chapitre au rapport entre reconnaissance et idéologie, et il ne le fait pas pour rien. Il part d'une objection contemporaine importante au concept de «reconnaissance», que nous pouvons résumer comme suit: la culture affirmative dans laquelle nous vivons - qui fait l'éloge de la reconnaissance sociale avant de contribuer à l'amélioration durable de l'autonomie des membres de cette culture - ne servirait-elle pas uniquement à reproduire des représentations conformes au système? Au lieu d'augmenter le pouvoir des sujets sociaux, cette reconnaissance ne les assujetit-elle pas? Ce n'est certainement pas une question mineure pour la thèse que Honneth développe dans une bonne partie de son travail philosophique, soutenu par une Théorie Critique construite par des bases intersubjectives. L'aspect élogieux du concept de reconnaissance qu'il soutient tant dans La société du mépris (2008) que dans La lutte pour la reconnaissance (2013) passe par une réponse à ce «scepticisme théorique» qui risque de definir l'auteur comme un simple idéologue. Tout l'effort de ce chapitre La reconnaissance comme idéologie sera de tenter de refuter la thèse selon laquelle «les individus sont poussés à adopter, au travers de processus de reconnaissance mutuelle, un rapport à soi spécifique qui les incite à assumer de leur plein gré des tâches et des devoirs servant la société» (HONNETH, 2008, 245-246). Si les auteurs contemporains de Honneth (comme Markell, Olivier et Verweyst) reprennent cette thèse, la cible de cette réfutation est la théorie de l'idéologie de Louis Althusser. C'est le philosophe français qui, déjà dans les années 1960 et 1970, faisait «de la pratique de la reconnaissance publique le mécanisme standard de toutes les formes de idéologie» (HONNETH, 2008, 246).

De ce fait, le recours à Rancière n'est pas fortuit dans cet article, d'autant plus que, déjà en 1974, dans La leçon d'Althusser, ce contemporain de Honneth s'opposait à la théorie de l'idéologie du maître. Souligner le parallèle entre ces formes d'opposition au projet althusserien peut nous aider à penser une discussion Rancière-Honneth capable d'amener à comprendre critiquement le concept de reconnaissance aujourd'hui, de même que les apports des deux philosophes à la discussion majeure de la politique contemporaine ${ }^{2}$. Dans ce sens, nous développerons le présent travail en trois étapes: la première sera consacré à la présentation et à la 
critique adressée par Honneth à Althusser; la deuxième à la critique de Rancière à l'encontre de la théorie de l'idéologie althusserienne; et la dernière, à la proximité et à l'éloignement entre les deux critiques et aux projets philosophiques des deux auteurs.

\section{La critique de Honneth à Althusser}

Pour aborder le concept althussérien d'idéologie, Honneth part de la constatation que le terme «subjectivation» possède, en français, un double sens, qui permet I'élaboration conceptuelle d'Althusser. «Subjectivation» peut signifier un processus actif - devenir sujet - aussi bien qu'un processus passif - s'assujettir. Selon Honneth, c'est ce qui permet à Althusser de penser que les êtres humains ne deviennent sujets, dans le sens d'une prise de conscience de leurs propres droits et responsabilités, qu'en étant assujettis à un système de règles pratiques et d'attributions qui leur confèrent une identité sociale.

Selon Honneth, cette caractérisation enlève au concept de «reconnaissance» toute sa connotation positive, puisque reconnaître quelqu'un signifie l'amener à adopter exactement le rapport à soi qui convient au système établi d'attente de comportements. D'une manière plus descriptive que critique, selon Honneth, Althusser ne porte aucun jugement normatif sur le processus de reconnaissance. C'est pourquoi une théorie critique de la société représente chez Althusser un grand défi, à savoir celui de trouver un fondement normatif capable d'assurer que la reconnaissance sociale ne possède pas uniquement comme fonction le maintien de la domination sociale. Le sens péjoratif auquel le mot «idéologie» est souvent associé découle précisément de ce fait qui tend à considérer que toutes les formes de reconnaissance sont fausses et injustifiées, puisqu'elles ne servent pas à affirmer l'autonomie personnelle, mais à produire des attitudes conformes à la domination.

Honneth considère cependant fausse l'accusation qui cherche à imputer à la théorie de la reconnaissance l'impasse sur ces phénomènes négatifs d'assujettissement et de domination. Selon l'auteur, toute cette problématique doit son élan critique au point de départ des manifestations sociales d'absence ou de déficit de reconnaissance. Ce sont les pratiques d'humiliation et les atteintes à la dignité humaine, privant les sujets d'une forme légitime de reconnaissance, donc d'autonomie, qui ont engendré cette vision critique du processus même de reconnaissance. Par ailleurs, nous dit Honneth, cette manière de formuler la question montre justement que la «reconnaissance» a toujours été traitée comme opposée aux pratiques de domination et d'assujettissement; c'est-à-dire, d'une façon 
positive. Cependant, ce «présupposé» de non-domination de la «reconnaissance» ne se prête pas à d'autres développements à partir de la pensée d'Althusser, car les formes de reconnaissance qui l'intéressent sont précisément celles qui deviennent effectives en tant que moyens de domination sociale ${ }^{3}$.

Dans un premier mouvement d'opposition à thèse idéologique de la reconnaissance, Honneth se sert de l'argument du temps. D'après lui, le choix des exemples et leur description ne peuvent pas être perçus comme idéologiques si on ne tient pas compte qu'ils sont le résultat d'un jugement moral qu'il n'est possible de faire qu' à partir de la perspective d'un présent moralement plus avancé. C'est-à-dire que c'est parce que nous pouvons regarder vers le passé, avec les caractéristiques du présent et de ses développements, que nous pouvons voir le discours sur les femmes et sur la guerre, ainsi que la reconnaissance des individus y impliqués, comme étant idéologiques. Honneth va même jusqu'à soutenir que «dès que nous nous plaçons dans des contextes passés, il devient infiniment plus difficile de distinguer entre une forme de reconnaissance fausse et "idéologique"» (HONNETH, 2008, 249). Si cette constatation du rôle du temps dans la considération d'une reconnaissance sociale comme étant «idéologique» est vrai, de même est vrai le discours également retrospectif de Honneth, qui essaie d'assigner au passé ce qu'il pouvait ou ne pouvait pas être. A moins de comprendre cette position comme une hypothèse critique de travail, c'est-à-dire, comme une tentative de montrer une autre forme de regard sur la question idéologique - sans, en principe, prendre aucune position sur les limites ou les ouvertures du passé - la position de Honneth n'a pas de sens. C'est dans cette perspective que nous entendons les questions et les affirmations «rhétoriques» que Honneth destine aux exemples qu'il cite (HONNETH, 2008, 249).

Son but est de montrer le caractère extrêmement délicat de I'identification du contenu idéologique des formes de reconnaissance. Ce n'est que d'un point de vue phénoménologique que Honneth se propose de penser cette question du rapport entre reconnaissance et idéologie. C'est pour cela qu'il affirme que «c'est seulement lorsque les sujets concernés se sont eux-mêmes révoltés contre une pratique dominante de la reconnaissance que nous disposons, pour la période en question, de premiers points d'appui nous permettant de parler de pure idéologie» (HONNETH, 2008, 249-250). Honneth, cependant, reste attaché, même si ce n'est que partiellement, à la structure «idéologie» versus «vérité», «idéologie» versus «science», qui a tellement caractérisé le travail d'Althusser, et qui deviendra une partie de la critique de Rancière. Honneth nous dit que cette difficulté à traiter les cas passés diminue normalement au fure et à mesure que la distance temporelle augmente; car nous disposons de plus de «critères admis de manière générale pour opérer rétrospectivement une distinction entre des formes idéologiques et des 
formes de reconnaissance revendiquées sur un plan moral» (HONNETH, 2008, 250). Donc, au fur et à mesure que le temps passe, plus la science historique a la possibilité de distinguer l'idéologique du moral, dans chaque cas. Rien de plus althussérien, donc.

Toutefois, Honneth sait aussi que cette distinction n'est pas tout à fait satisfaisante, car nous ne disposons pas entièrement d'indices empiriques capables de montrer que les individus concernés désapprouvent euxmêmes certaines pratiques de reconnaissance répressives, restrictives ou normalisatrices. La question théorique reste, de ce fait, toujours ouverte. Selon Honneth, la raison de cette difficulté réside dans le fait conceptuel qu' un acte de reconnaissance décrit toujours la manifestation publique d'une «valeur ou d'une performance censées revenir» à une personne ou à un groupe social. La question qui apparaît alors à Honneth est: «comment, à I'heure actuelle, des formes de manifestation publique d'une valeur sociale, en l'occurrence de la reconnaissance, peuvent elles néanmoins avoir les traits de la domination?»(HONNETH, 2008, 250). C'est justement à cette question que La reconnaissance comme idéologie essaie de répondre.

\section{L'opposition à Althusser: la distinction entre les formes idéologiques et les formes justifiées de la reconnaissance}

Pour le première partie de son argumentation, Honneth affirme que le concept de reconnaissance d'Althusser possède un sens unidimensionnel, sans distinguer le vrai du faux, le justifié de l'idéologique. Selon Honneth, pour Althusser toute forme de reconnaissance a un caractere idéologique, du simple fait que solliciter ou interpeller quelqu'un impose toujours une unité imaginaire. Pour s'opposer à Althusser, Honneth a donc besoin de distinguer les formes idéologiques des formes justifiées de la reconnaissance; ce qui implique d'assumer un caractère positif pour la reconnaissance. Après une briève description historique du concept, se reportant surtout à l'emploi hégélien du terme, Honneth parvient à une première assignation concernant la reconnaissance: «il faut tenir en compte que la reconnaissance doit renvoyer à un acte moral ancré dans le monde social en tant qu'événement quotidien» (HONNETH, 2008, 252). Cette réference au jeune Hegel d'Iéna permet à Honneth d'aborder une certaine grammaire morale des conflits sociaux. Il soutient cette thèse à partir de quatre grandes prémisses. La première: la reconnaissance «est l'affirmation de qualités positives de sujets humains ou de groupes» (HONNETH, 2008, 253). La deuxième: «un acte de reconnaissance ne peut se réduire à de purs mots ou expressions symboliques puisque seuls les comportements correspondants lui donnent la credibilité qui est normativement importante 
pour le sujet reconnu» (HONNETH, 2008, 253). La troisième: les actions de reconnaissance constituent un phénomène distinct dans le monde social qui «ne doit pas être compris comme un sous-produit d'une action orientée dans un autre sens, mais comme l'expression d'une intention autonome» (HONNETH, 2008, 253). Quatrième: «la "reconnaissance" représente un concept générique englobant différentes sous-variantes» (HONNETH, 2008, 253). À partir de toute cette clarification théorique, Honneth peut definir la «reconnaissance» comme étant «le caractère générique des différentes formes prises par une attitude pratique dont l'intention première consiste en une certaine affirmation du partenaire d'interaction» (HONNETH, 2008, 254). Il convient également de rappeler ici l'influence de la psychologie sociale de Mead sur l'élaboration conceptuelle de Honneth.

Ainsi, d'une façon différente du philosophe français, Honneth propose un caractère positif du concept de reconnaissance. Cette caractéristique permet aux destinataires de s'identifier à leurs propres qualités et à accéder de ce fait à davantage d'autonomie. Le défi d'une telle explication, cependant, est de montrer dans quelle mesure la reconnaissance est un acte attributif ou un acte réceptif; car, dans le premier cas, la reconnaissance serait un acte d'attribution par lequel une autre personne attribue une qualité, nouvelle et positive; tandis que dans le second, au contraire, cet acte suit le modèle d'une perception par laquelle la qualité en question d'une personne ne se manifeste que publiquement, ou est renforcée d'une façon secondaire. En tant qu'acte attributif, la reconnaissance consisterait en un accord ou un statut à ajouter à un sujet concerné qu'il ne pouvait pas avoir auparavant. Mais, en tant qu' acte réceptif, il s'agirait d'une forme précise de perception d'un statut indépendant qui existerait préalablement. Honneth nomme le premier cas «reconnaissance productive», et le deuxième «reconnaissance reproductive». L'auteur voit cependant dans cette dichotomie un problème suscetible de soutenir, peut-être, les difficultés de la caractérisation idéologique d'Althusser, car ni la simple attribution depuis l'extérieur ni la simple reproduction de l'externe ne donneraient pas au concept de «reconnaissance» les implications morales, évaluatives, d'autonomie du sujet, que Honneth apprécie tellement.

L'inconvénient de la reconnaissance productive est le fait que si une attitude de reconnaissance d'une autre personne pouvait attribuer au sujet immédiatement des qualités positives, alors il n'y aurait aucun critère interne pour juger du caractère juste ou approprié de ce genre d'attribution. Tout ce qui émane d'un acte d'attribution serait alors considéré une capacité ou un statut de la personne. Le risque ici serait de tomber dans une approche sociologique, que Honneth appelle «étiquettage». La 
reconnaissance reproductive, toutefois, n'offre pas, quant à elle, de meilleures conditions, car prétendre que l'attitude de reconnaissance suppose une réaction «juste» aux qualités d'une personne ou d'un groupe implique la supposition de l'existence objective de valeurs; ce que Honneth ne parvient pas à concevoir. Cela reviendrait à présupposer des qualités objectives et fixés par lesquelles les individus pourraient percevoir, et simplement reproduire. Honneth s'oppose à ce réalisme moral qui concevrait les valeurs de façon pré-déterminée. Il essaie de les penser d'une manière historique et historiquement transformables. Le danger de cette conception, selon Honneth, c'est que les qualités que nous pouvons reconnaître chez les autres ne peuvent êtres perçues qu' à I'horizon de l'expérience d'un monde vécu spécifique, ce qui nous ferait tomber dans un particularisme culturel, et donc dans une sorte de relativisme, incompatible, dans la vision de l'auteur, avec «les objectifs normatifs du concept de reconnaissance» (HONNETH, 2008, 257). On perçoit alors la motivation universalisatrice du projet de Honneth, encore redevable, dans une certaine mesure, de son affiliation habermasienne.

Cependant, plus près du modèle de réception que de celui d'attribution, Honneth essaie d'indiquer un parcours selon lequel cette approche pourrait éviter les difficultés qu'elle met en relief. Pour ce faire, il faudrait doter le réalisme moral du modèle de réception d'une solide conception de progrès. Sans entrer dans les détails de ce processus, l'auteur semble néanmoins le penser comme une forme de savoir réflexif selon lequel nous nous orienterions dans le monde à partir des expériences vécues des autres. À partir de la socialisation, nous pouvons apprendre à considerer de manière rationnelle avec d'autres êtres humains les qualités que nous pouvons reconnaître chez les autres. Nous pourrions, peut-être, dire qu'il s'agit là d'une façon de sauver le présupposé objectif des valeurs à partir des apprentisages historiques de la société. Cependant, une telle conception progressiste peut aussi présenter des difficultés, comme le montrera Rancière. Pour Honneth, cependant, ces problèmes semblent ne pas faire surface.

\section{L'hypothèse de Honneth}

Honneth ouvre la deuxième partie de sa contre-argumentation à la thèse idéologique en assumant explicitement la thèse que, jusqu'alors, il avait à peine ébauché, à savoir celle d'un réalisme moral modéré. C'est-àdire la thèse d'une «reconnaissance reproductive», ajoutée au concept de progrès (HONNETH, 2008, 260). 
Avec cette définition, Honneth cherche à se mettre dans une position de différence «majeure avec la proposition d'Althusser» (HONNETH, 2008, 260), car pour celui-ci toute forme de reconnaissance représente nécessairement une forme d'idéologie. Pour lui, selon les mots de Honneth «la simple attribution d'un statut social est déjà une pratique idéologique, indépendamment du contenu de ce qui est adressé aux sujets, car elle produit simultanément une illusion d'unité et d'identité et une disposition à accepter les attentes de comportement correspondantes» (HONNETH, 2008, 261). Pour Honneth, cependant, la question part «de la possibilité d'une forme approprié et rationnelle de reconnaissance consistant à faire valoir publiquement et de manière performative certaines qualités préexistantes de l'être humain» (HONNETH, 2008, 260). Honneth propose donc une restriction de sens moral à la thèse althusserienne. Toutes les attributions ne seront pas comprises comme «reconnaissance»; seulement celles préexistantes, qui ont une valeur et qui sont réalisés publiquement. La critique de Honneth à Althusser est donc une critique morale. Althusser ne serait pas parvenu à séparer les formes justifiées de «reconnaissance» des formes idéologiques. Si tout est idéologique, le risque de relativisme revient en force, et c'est ce qui préoccupe Honneth.

L'aspect moral de la reconnaissance, sur lequel le philosophe allemand attire fortement l'attention, apparaît seulement à partir de ce qu'il appelle la «limitation de l'égocentrisme» (HONNETH, 2008, 261). C'est une idée qu'il emprunte au concept de respect de Kant. Pour Honneth, «reconnaître quelqu'un signifie percevoir en lui des qualités qui nous incitent de manière intrinsèque à nous comporter non plus de manière égocentrique mais conformément aux intentions, besoins ou désirs de cette autre personne» (HONNETH, 2008, 261). Et cette valeur ne doit pas être comprise d'une façon relativiste, c'est-à-dire les valeurs de chacun; mais comme des «valeurs dignes d'êtres reconnues chez les sujets humains» (HONNETH, 2008, 261). L'universalisme de Honneth apparaît ici.

Face à la discussion sur l'aspect idéologique de la reconnaissance, Honneth peut, maintenant, soutenir la thèse que l'on ne peut pas réduire le spectre d'énoncés publiques de valeur et de représentation des individus à un rôle simplement idéologique, car les classifications évaluatives de notre monde ne peuvent pas être remplacés, dans la plupart du temps, par des considérations idéologiques. Les idéologies, nous dit Honneth, doivent potentiellement tendre à exprimer une valeur positive d'un sujet ou d'un groupe social, afin de donner aux individus la possibilité d'établir un rapport à soi d'une façon positive, en acceptant volontiers la tâche qui leur est destinée par l'idéologie. Dans ce sens, Honneth pense que des formes péjoratives d'identification d'individus ou de groupes, comme le racisme, la 
misogynie ou la xénophobie, ne peuvent, en aucun cas, assumer le rôle d'idéologies de la reconnaissance, car elles déforment l'image de soi des sujets auxquels elles s'adressent. Les idéologies ne servent donc pas à une reconnaissance qui conduirait à l'exclusion; mais, au contraire, elles contribuent à l'intégration. Elles doivent donc être «crédibles» aux yeux des individus concernés. Si les destinataires ne se voient pas dans les énoncés de valeur proposés par l'idéologie, il n'y a aucun caractère de performance dans un tel usage, ce qui est un aspect sur lequel Honneth attirait déjà l'attention au début de sa discussion. De la même façon, il affirme que les énoncés doivent être réalistes, c'est-à-dire, qu'ils doivent se destiner d'une façon significante aux attitudes ou vertus réelles de leurs destinataires. Enfin, les énoncés doivent êtres rationnels, dans le sens d'une rationalité historique qui, à partir du «vocabulaire évaluatif du présent» (HONNETH, $2008,263)$, peut être assumée comme vérité. Des qualités discréditées du passé, «démodées», ne peuvent pas non plus offrir la performance voulue. Par exemple, faires compliments aujourd'hui à une femme pour ses vertus de maitresse de Maison lui offre peu de raisons pour s'identifier à cet énoncé.

Considérer les idéologies comme irrationnelles signifierait ne pas comprendre pourquoi elles sont effectives, pourquoi elles ont un effet de performance sur les sujets concernés. C'est ainsi qu'il distingue les «idéologies excluantes» et des «idéologies de reconnassaince». Alors que les premières font «éclater I'horizon perceptif d'évaluation en vigueur en rendant des sujets sociaux aveugles à certaines qualités d'autres groupes sociaux» (HONNETH, 2008, 265), les deuxièmes «élargissent uniquement les qualités évaluatives que nous avons appris à percevoir chez d'autres êtres humains en accentuant une signification nouvelle dont le propre est cependant de faire advenir, lorsqu'elle est reprise avec succès, un rapport à soi adapté et fonctionnellement conforme» (HONNETH, 2008, 265). C'est cette distinction optimiste de Honneth qui lui permet de penser que ces «idéologues rationnelles» ne peuvent pas ne pas se «référer sémantiquement aux principes de l'amour, de l'égalité juridique ou de la juste récompense des prestations» (HONNETH, 2008, 266); c'est-à-dire, à la façon dont il pense la reconnaissance.

\section{Le test de l'hypothèse}

Pour compléter sa contre-argumentation à Althusser, Honneth fait appel à un exemple d'une nouvelle domination, dans le monde du travail, comme une sorte de test de ses hypothèses de travail. L'exemple vient de la littérature de gestion des entreprises qui n'appelle plus les travailleurs 
«salariés» ou «main-d'-oeuvre», mais «travailleurs-entrepreneurs». Honneth montre qu'il est facile de voir dans cette façon de s'adresser aux travailleurs un sens «idéologique», comme une simple façon de maquiller les effets de flexibilisation et dérégulation néolibérales du capitalisme pour qu'ils assument volontairement les exigences actuelles du monde du travail. Mais, s'il y a un sens dans le fait d'appeler cet exemple «idéologique», il doit l'être dans le sens que Honneth lui donne, c'est-à-dire, d'une façon que «les sujets auront de bonnes raisons évaluatives de s'attribuer la nouvelle qualité, de manière à parvenir aussi, le cas écheant, à un sentiment accru de leur propre valeur ou de leur estime d'eux-mêmes» (HONNETH, 2008, 270). Cette nouvelle qualité doit donc, au moins, être «perçue par les employés comme suffisamment "crédibles" pour qu'ils puissent la reprendre à leur compte» (HONNETH, 2008, 271).

Selon Honneth, cette crédibilité se fonde sur deux composantes. D'un côté, il s'agit de la composante évaluative, que nous avons démontré jusqu'ici; et, d'un autre côté, il s'agit de ce qu'il appelle la composante «matérielle»; c'est-à-dire, des comportements appropriés ou des dispositions institutionnelles correspondantes. Cette deuxième composante agit sur la crédibilité à partir du fait que la matérialité donne de la force à des attitudes de reconnaissance (HONNETH, 2008, 272).

C'est justement cette composante matérielle qui permet à Honneth d'offrir une solution aux problèmes complexes consistant à différencier les formes idéologiques des formes justifiées de reconnaissance. Dans l'exemple du test de Honneth, ce qui rend la reconnaissance des «travailleurs-entrepreneurs» ou «entrepreneurs d'eux-mêmes» idéologique - et non justifiée - c'est le fait que la promesse de reconnaissance du plan évaluatif ne s'accompli pas matériellement dans les dispositions institutionnelles. Au contraire, nous dit Honneth, «les destinataires sont contraints, dans des conditions de travail inchangées, de simuler des motivations, de la flexibilité et des compétences» (HONNETH, 2008, 273). Si elles peuvent être considérés rationnelles au plan où leur vocabulaire se réfère au domaine historiquement modifiable des raisons évaluatives, elles ne peuvent pas l'être dans le sens qu'elles ne sortent pas du simple plan symbolique pour se concrétiser dans des instances matérielles. Même si Honneth est conscient que cette argumentation se fonde seulement sur un critère de temps qui peut être employé contre lui, puisque la matérialité d'un acte de reconnaissance peut toujours être attendue dans un avenir. Mais, même dans ce cas, il considère que «le critère de réalisation matérielle est utile surtout pour examiner si, lors de la transformation d'un aspect de la reconnaissance, on assiste dans les faits à un accroissement du pouvoir régulateur» (HONNETH, 2008, 275). A tous ces modèles institutionnels de 
distinction évaluative, auxquels il manque la composante matérielle, le qualificatif «idéologique» est bien approprié.

Après toute cette «hygiène» conceptuelle, Honneth parvient à proposer une façon d'essayer d'identifier des formes justifiées de reconnaissance qui s'opposeraient à une forme idéologique. Mais, cette proposition de Honneth est-elle soutenable? C'est que nous aimerions vérifier maintenant, en la confrontant à une discussion avec une autre critique à la même théorie de l'idéologie d'Althusser, à savoir, celle de Rancière.

\section{La critique de Rancière à I'encontre de la théorie de I'idéologie althusserienne}

Les critiques de Rancière à son maître de l'ENS, publiées en 1974 dans La leçon d'Althusser, commencent déjà à prendre forme en 1969, lors d'un cours à Paris VIII que l'auteur consacre aux textes de Marx, sur l'idéologie, et qui débouchent sur le marxisme althussérien, et plus précisément sur l'influence de cette interprétation dans le contexte universitaire post-Mai 68. Ce premier texte, paru d'abord dans un recueil de textes sur Althusser publié en Argentine en 1969, et qui ne paraît en France qu'en 1973 - dans la revue L'Homme et la Société -, constitue aujourd'hui l'appendice de la nouvelle édition de La leçon d'Althusser.

Dans ce texte, dont le titre est Sur la théorie de l'idéologie: politique d'Althusser, Rancière part d'une synthèse de la spécificité de la thèse althussérienne pour montrer que son interprétation de Marx ainsi que la politique révisionniste de justification théorique "antigauchiste" ont un rapport théorique et politique réel. Les deux thèses fondamentales de la théorie d'Althusser sur l'idéologie sont, selon Rancière, les suivantes: «1. L'idéologie a dans toute société - divisée ou non en classes - une fonction première commune: assurer la cohésion du tout social en réglant le rapport des individus à leurs tâches. 2. L'idéologie est le contraire de la science.» (RANCIÈRE, 2011, 216).

La première thèse est celle que Honneth a en tête pour sa critique d'Althusser, laquelle nous pourrions traduire en termes de reconnaissance, même si Rancière n'emploi pas ce mot. La deuxième, cependant, n'a semble pas être traitée explicitement par Honneth, même si elle l'aide à formuler sa réponse «scientifique» au problème de l'idéologie.

Rancière décrit la première thèse comme étant celle qui vise à critiquer les idéologies de la désaliénation, c'est-à-dire, les thèses qui pensent que la fin de l'aliénation capitaliste serait la fin de la mystification de la conscience, un monde «transparente» dans les rapports homme- 
nature et homme-homme. Même si Honneth ne parle pas de désaliénation, nous pourrions, peut-être, penser son architecture morale visant une reconnaissance justifiée - parallèle à la reconnaissance - comme une sorte de revendication de transparence. C'est à cette tradition d'une idéologie de la transparence - qui remonte à Saint Paul - qu'Althusser aurait opposé «I'opacité nécessaire de toute structure sociale à ses agents» (RANCIÈRE, 2011 , 217). Et ceci parce que l'idéologie se situe dans toute totalité sociale lorsqu'elle fournit «le système de représentations qui permettent aux agentes de la totalité sociale d'accomplir les tâches déterminées par cette structure» (RANCIÈRE, 2011, 217). Sans une certaine opacité les tâches escomptées par la structure ne seraient pas accomplies, ou, du moins, pourraient-elles être reportées. Ce n'est donc que l'idéologie qui pourra assurer cette réfutation du rapport entre les individus et leurs tâches. La différence d'Althusser, dans ce cas, consiste à généraliser l'idéologie pour n'importe quel type de société. Dans les sociétés sans classes, aussi bien que dans les sociétés de classes, l'idéologie aurait la même fonction: assurer ce lien entre des hommes. Si cette affirmation provoque une certaine surprise chez Honneth, elle a aussi le même effet chez Rancière. Celui-ci, comme son confrère allemand, cherchera, d'une façon ou d'autre, à sortir de cet enfermement. Pour des raisons différentes, plus politiques que morales, Rancière cherchera à expliquer les problèmes de ces thèses fondamentales de la théorie de l'idéologie d'Althusser.

Le premier problème découle du fait que, avec cette généralisation, Althusser avance le concept d'idéologie avant que n'intervienne le concept de lutte de classes. Le concept primordial du marxisme finit par «surdéterminer la fonction première de l'idéologie» (RANCIÈRE, 2011, 217); c'est l'idéologie qui est le concept de base de toute explication politique. Cette généralisation, engendre un problème, selon Rancière, à mesure qu'il fait avancer certains concepts pour rendre compte de cette tâche, et qui ne se reportent pas à la tradition marxiste, mais plutôt à des sociologues du type comtienne ou durkheimnienne. «Lien», «cohésion», ou «tout social» sont des termes beaucoup plus proches de cette tradition qui se soucie «des systèmes de représentations qui assurent ou désagrègent la cohésion du groupe social» (RANCIÈRE, 2011, 219) plutôt que d'une tradition qui pense que «toute I'histoire de I'humanité est celle de la lutte des classes» (RANCIÈRE, 2011, 219). La réponse de Honneth au problème de la reconnaissance semble suivre la même piste sociologique, même si c'est sur des traces différentes de celle d'Althusser.

Rancière, quant à lui, se pose la question de savoir comment son maître a pu définir comme fonction de l'idéologie celle d'«assurer la cohésion sociale en général» (RANCIÈRE, 2011, 219) après avoir considéré, 
avec le marxisme, la lutte de classes comme le moteur de l'histoire. La réponse de Rancière passe par la constatation que ce déplacement d'Althusser est dû à une certaine concession au modèle d'analyse traditionnel de la religion. Lorsqu'il pense que dans les sociétés primitives sans classes - la religion constituait déjà une première forme générale d'idéologie, Althusser finit par se trouver face à une analyse sociologique qui pourrait bien plaire à Durkheim ou à Comte. Rancière questionne alors Althusser en inversant l'analyse: «penser l'idéologie en général avant de penser la lutte des classes, n'est-ce pas nécessairement le penser sur le modèle de l'analyse traditionnelle de la religion, celle d'une sociologie héritière du discours métaphysique sur la société?» (RANCIÈRE, 2011, 219). C'est justement ce recours métaphysique que Rancière essaiera de démontrer comme étant la caractéristique des deux thèses d'Althusser: celle qui généralise l'idéologie à toute sorte de société et celle qui distingue idéologie et science. Nous pourrions dire que ce qui est en jeux dans la critique de Rancière, c'est un certain platonisme d'Althusser.

En ce qui concerne la métaphysique de cette première thèse, Rancière montre qu'Althusser transforme le coexistence de ces deux conceptualisations hétérogènes - le matérialisme historique et la sociologie bourgeoise du type durkheimnienne - en articulation. Dans la manière de voir de Rancière, cela implique une subversion de l'idéologie en deux sens. Pour le premier sens, l'idéologie n'est plus définie en termes marxistes, mais en termes d'une sociologie générale. La théorie marxiste finit par «se surposer à cette théorie sociologique de l'idéologie comme théorie d'une surdétermination propre aux sociétés de classes» (RANCIÈRE, 2011, 220). De ce fait, les concepts qui définissent la fonction de l'idéologie dans une société de classes dépenderont des concepts de cette sociologie générale; du concept de «cohésion sociale», par exemple. Le deuxième sens de cette subversion de l'idéologie passe par la revendication de cette sociologie générale comme un niveau de la théorie marxiste de l'idéologie, même si celle-ci n'a rien à y dire. La question, dans ce deuxième sens, est d'utiliser les concepts marxistes qui définissent une société de classes pour definir la société en général.

Le problème de cette subversion, c'est que, pour la tradition marxiste, la détermination d'une totalité par sa structure se fonde simplement sur les rapports de production qui caractérisent un mode dominant de production. Rancière nous dit que «la manifestation/dissimulation de la structure ne signifie pas une opacité de la "structure sociale en général", elle est l'efficace des rapports de production» (RANCIÈRE, 2011, 222). Extrapoler cette efficacité de la structure au-delà des sociétés de classes la transforme en «un concept parfaitement indéterminé - ou bien alors déterminé comme 
le substitut d'une figure traditionnelle de la métaphysique: malin génie ou ruse de la raison» (RANCIÈRE, 2011, 222). La sauvegarde métaphysique agit, de ce fait, chez Althusser, de la même façon qu'elle agit chez Descartes ou chez Hegel. La conséquence en est que l'idéologie n'est plus pensée comme un lieu d'une lutte, par référence à deux antagonistes, mais comme une totalité dans laquelle est un élément naturel. C'est le fait de rendre I'idéologie quelque chose de naturel qui lui confère un statut métaphysique, comme une structure qui fonde toute réalité.

Si elle n'a pas été au début considérée comme étant un champ de lutte, l'idéologie «sera subrepticement devenue un des partenaires de la lutte» (RNCIÈRE, 2011, 224). Rancière nous dit alors que la lutte de classes, oubliée au début de l'analyse d'Althusser, «réapparaîtra sous une forme fantastique, fétichisée, comme lutte de classes entre l'idéologie (arme de la classe dominante) et la science (arme de la classe dominée)» (RANCIÈRE, $2011,224)$. La première thèse fondamentale d'Althusser rejoint, ainsi, la deuxième. L'extrapolation $\mathrm{du}$ concept d'idéologie comme forme d'explication de toute société - avec ou sans classes - fait apparaître une seule issue pour Althusser: I'opposition idéologie et science. Dans la vision d'Althusser, ce sera la science qui nous permettra d'échapper à la domination imposée par le système de représentation idéologique. Dans une autre synthèse des idées d'Althusser, Rancière propose un schéma qu'il appelle «logique de l'oubli» dans lequel le système de représentations n'est donc pas un système des illusions nécessaires aux sujets historiques, mais acquiert une fonction supplémentaire de maintenir les individus à la place déterminé par la domination de classe.

La question de cette thèse concerne donc le passage de l'idée de la domination d'une idéologie vers la domination de l'idéologie. C'est pour cette raison que la science apparaîtra comme une réponse. C'est parce que l'idéologie est une représentation fausse - qui ne permet pas aux hommes de connaître le système social dans lequel ils sont insérés - que la seule solution d'Althusser pour une subversion de cette domination sera de faire appel «à l'Autre de I'Idéologie, c'est-à-dire la Science» (RANCIÈRE, 2011, 225). Et les capitales n'y sont pas un hasard. Rancière a à l'esprit I'histoire de la métaphysique, et la référence explicite à Platon (Le Sophiste) y est évidente (RANCIÈRE, 2011, 225-226).

Rancière est assez clair dans son argumentation. Il nous dit que ce placement du concept d'idéologie dans le lieu classique de I'histoire de la métaphysique, comme l'Autre de la Science, peut seulement être compris si I'on comprend l'absence (ou I'oubli), au début du travail d'Althusser, de penser l'idéologie comme le lieu d'une lutte, d'une lutte de classes. C'est parce que l'idéologie ne s'insère pas dans le jeux de structure de la lutte de 
classes, mais parce qu'elle le «saute», qu'elle peut apparaître comme un espace homologue de l'Autre de la Science; tel que l'avait pensé l'histoire de la métaphysique depuis l'opposition epistème versus dóxa. L'idéologie ne peut donc être surpassée que par le travail de la Science, et non plus par le travail de la lutte de classes. Ou, mieux, le travail de la lutte de classes se résume au travail de mettre la science en route. C'est cette opération qui est en jeux avec Althusser, et c'est contre le projet d'«éclaircissement», de «conscientisation» que Rancière se lance.

A cette époque-là, le philosophe n'avait pas encore connaissance des archives ouvrières, et connaissait encore moins le pédagogue Joseph Jacotot, qui lui permettront, plus tard, d'établir un contre-projet plus effectif à la différence d'intelligences apparaissant dans les thèses d'Althusser. Mais, Rancière possède déjà une bonne connaissance de la tradition marxiste capable de fournir une réponse consistante à l'issue proposée par le philosophe de I'ENS. En 1969, Rancière suggère que l'erreur aurait été de confondre une distinction modale du marxisme et une distinction réelle. La distinction entre «division technique» et «division sociale» du travail qui correspondent à deux façons de conceptualiser le même processus, c'est-à-dire que «les functions qui assurent la reproduction technique du procès sont les mêmes qui déterminent sa reproduction sociale» (RANCIÈRE, 2011, 229) - est prise par Althusser comme une distinction réelle qui établit des lieux et des fonctions spécifiques pour l'une ou l'autre division. Dans une référence à l'article Problèmes étudiants d'Althusser, publié en 1964 dans La Nouvelle Critique, Rancière montre comment le philosophe de l'ENS établit cette correspondance (RANCIÈRE, 2011, 229).

La formulation énigmatique, comme la définit Rancière, nous amène à essayer de comprendre comment il serait possible de définir les besoins, exclusivement techniques d'un mode de production qui seraient indépendants de leur finalité sociale, et qui, à leur tour, impliqueraient la reproduction de formes de division en classes et de la domination de classe. La solution d'Althusser passe par l'exemple qu'il donne de I'Université comme institution faisant partie de toute société moderne. La division technique du travail, avant de correspondre aux exigences d'un mode de production spécifique correspond aux besoins techniques d'une société moderne. Tout se passe donc «comme si» on pouvait «définir un certain nombre de places et de fonctions nécessaires d'une société moderne en général, en fonction du seul niveau de développement des forces productives» (RANCIÈRE, 2011, 230). C'est-à-dire que la division technique concernerait - à un niveau de développement donné des forces productives - la modernité, la division sociale concernant la reproduction des rapports de production déterminé dans cette société. 
Cette caractérisation permet alors à Rancière de proposer un lieu ébauché au début de son article, à savoir celui qui lie à l'interprétation althusserienne de l'idéologie de Marx la politique révisionniste de justification théorique «antigauchiste». C'est le concept de division technique du travail, tel que le définit Althusser, qui permet, selon Rancière, «la simple justification des mots d'ordre révisionnistes fondés sur les notions de "besoins réels de la nation", "besoins réels de l'économie", "modernisation", etc.» (RANCIÈRE, 2011, 231). Rancière cite même I'usage que le parti communiste français a fait de cette thèse, passant d'une dialectique marxiste à un «éclectisme de type proudhonien qui distingue le bon et le mauvais coté des choses» (RANCIÈRE, 2011, 231). Ce maniquéisme, auquel la notion d'idéologie est restée depuis associée, découle de cette interprétation althusserienne, et de ce que Rancière appelle «la politique d'Althusser»; c'est-à-dire, les usages révisionnistes d'une «contre-révolution intellectuelle».

Dans une certaine mesure, il serait également possible aujourd'hui, plus de 50 ans après cette discussion, de percevoir les impacts de la politique d'Althusser sur les évolutions réelles du mouvement de gauche. Même dans un contexte sud-américain, comme celui du Brésil, les usages révisionnistes, «bien comportés», de la partie de la gauche qui faire appel aux «besoins réels de l'économie» ou à la «modernisation», semblent aussi justifier une action politique marquée par la redistribution et, non plus, par le dépassement d'un mode de production. Une contre-révolution intellectuelle a également conduit, dans notre scénario, à une approximation de la gauche aux idéaux néolibéraux, capable de produire une déception qui explique peut-être, au moins en partie, un certain retour au conservatisme. Comme nous dit Rancière en 1988, surpris par le vote expressif de Jean-Marie Le Pen à l'élection présidentielle: «là où le politique s'affaisse, où le parti des riches et des pauvres ne disent plus apparemment que la même chose - modernisation - [...] ce qui apparaît sur le devant de la scène n'est pas ce qu'on attendait: le triomphe de la modernité, mais le retour du plus archaïque, de ce qui précède tout jugement, la haine de I'autre» (RANCIÈRE, 1998, 54).

Il est clair qu'une explication de l'ordre de la psychologie ou de la sociologie est toujours possible, expliquant la haine par la frustration, comme si la haine venait de ce que je n'ai pas. C'est ce qui expliquerait la xénophobie européenne envers ceux qui «voleraient leur emploi». C'est aussi ce qui nous permet de voir dans le conservatisme brésilien un certain refoulement des acquis des autres, notamment des groups traditionnellement marginalisés, mais ce serait «s'autoriser à penser que l'affaire est seulement d'un retard dans la modernité, d'une survivance de 
la guerre dépassé des riches et des pauvres» (RANCIÈRE, 1998, 56). Ce serair blâmer le temps, car il n'y aurait pas eu assez de temps «pour que tous bénéficient des fruits de la croissance» (RANCIÈRE, 1998, 56). Ainsi, il y aurait une distinction entre les retardataires et les profitants, entre le vide et le plein, distinction qui servirait très bien l'explication savante d'Althusser.

La thèse du philosophe de l'ENS opère, dès lors, une double distorsion. La première, celle qui concerne le statut de l'idéologie, que Rancière pense avoir corrigé à partir du recours aux concepts «primitifs» du marxisme. Il lui reste alors à penser la deuxième distorsion, celle qui se fonde sur l'efficacité de la Science au service de la révolution. Le présupposé d'Althusser est que «le savoir scientifique a, en lui-même, une valeur subversive à l'égard de la domination bourgeoise» (RANCIÈRE, 2011, 233). $\mathrm{Si}$, d'un côté, Rancière reconnaît dans le champ propre aux thèses de Marx sur le socialisme scientifique une concession au fait que «l'affranchissement du prolétariat est impossible sans la théorie des conditions de cette libération, c'est-à-dire la science marxiste des formations sociales» (RANCIÈRE, 2011, 233), d'un autre côté, il pense que I'«on n'a aucun droît d'en induire une vertu révolutionnaire de la science en général» (RANCIÈRE, 2011 , 233). Rancière nous montre qu'une simple observation du champ de l'enseignement scientifique nous fournirait des donnés suffisantes pour justifier cette critique qu'il fait. La fonction réactionnaire de bon nombre d'enseignements due au manque de scientificité, mais aux structures de cet enseignement, au genre d'institution, aux mécanismes de sélection, etc. Dans ce sens, selon Rancière, «la science n'apparaît pas en face de l'idéologie comme son autre, elle apparaît à l'intérieur d'institutions et dans des formes de transmission où se manifeste la domination idéologique de la bourgeoisie» (RANCIÈRE, 2011, 234). La question pour les mouvements révolutionnaires, comme le posait Rancière en 1969, «n'est pas de leur poser des exigences de scientificité ou d'en appeler de ces pseudo-sciences à la scientificité idéale des mathématiques ou de la physique. Elle est d'opposer aux idéologies bourgeoises l'idéologie prolétarienne du marxisme-léninisme» (RANCIÈRE, 2011, 234-235).

Même si Rancière ne parle pas plus d'une idéologie marxiste-léniniste, il est intéressant de voir qu'il opposait aux idéologies bourgeoises, à un modèle d'explication de la réalité, non pas une explication «savante», mais un autre modèle, similaire, également idéologique, et donc, d'une certaine manière, «ignorant». Mais, il serait peut-être plus précis ici de dire idéologique, dans le sens d'en marge du système métaphysique vrai/faux. L'hypothèse de l'égalité des intelligences, que Rancière développera à partir de La Nuit des Prolétaires (1981) et, surtout, à partir de Le Maître Ignorant 
(1987), apparaît déjà en filigrane dans cette opposition à Althusser. Le point-clé de cette discussion semble être la considération que Rancière développe sur l'incarnation du savoir, sur les traces de Foucault. Rancière ne pense pas le savoir comme «un contenu qui peut être soit la science soit I'idéologie» (RANCIÈRE, 2011, 236). Ainsi pense la métaphysique, otage de ce que Rancière appelle «une théorie de l'imaginaire (conçu comme système de représentations qui séparent le sujet de la vérité)» (RANCIÈRE, 2011, 235). C'est à cette tradition épistémologique subjectiviste qu'Althusser est lié. Rancière, quant à lui, pense le savoir comme «un système où les "contenus" ne peuvent être pensés en dehors de leurs formes d'appropriation (acquisition, transmission, contrôle, utilisation)» (RANCIÈRE, 2011, 236). Dans la manière de voir de Rancière la séparation science/idéologie cache un nœud qui exprime la domination idéologique d'une classe; elle est ainsi une division inopérante pour analyser une situation concrète. Pour lui, il s'agit d'une ligne de partage simplement imaginaire.

Dans ce sens, selon Rancière, la distinction science/idéologie sert uniquement à justifier un être pur du savoir, et avec cela, à renforcer la «dignité» des porteurs du savoir, c'est-à-dire, des professeurs. C'est cette conséquence de la thèse d'Althusser qui saute aux yeux à la fin de l'article de Rancière. Il montre comment la théorie de l'idéologie de son maître sert publiquement à renforcer la vision qu'un projet émancipateur ne peut exister qu'à partir de la figure d'une hiérarchie qui sait, d'un professeur, d'un parti. Por démontrer cela, il montre comment la fonction pédagogique assume, dans l'article de son maître de I'ENS, un rôle fondamental dans son articulation conceptuelle, car elle a pour objet de transmettre un savoir déterminé à des sujets qui ne possèdent pas ce savoir. Donc la situation pédagogique repose sur la condition absolue d'une inégalité entre un savoir et un non-savoir.

C'est justement cette thèse de l'inégalité des intelligences, entre ceux qui savent et ceux qui ignorent, que Rancière contestera dans Le Maître Ignorant (1987). C'est à cette idée qu'un mouvement émancipateur dépend d'une pédagogie, fondée sur la différence des intelligences et sur la soumission de l'ignorance à la science, de la masse au parti, que Rancière s'opposera à partir de ses travaux sur les archives ouvriers du XIXe siècle. Cette vision «progressiste» est, dans sa manière de voir, seulement une continuation d'un mouvement abrutissant, et non pas émancipateur. Ce que la position d'Althusser révèle, dans sa cohérence avec la tradition métaphysique classique, c'est le prolongement de la «modestie propre à la tradition "épistémologique"» qui considère cette question comme étant «produite par la demande même de la science» (RANCIÈRE, 2011, 241). 
C'est-à-dire, depuis le début de la tradition métaphysique, au moins à partir de Platon, c'est toujours un intérêt «épistémologique» qui assure la dispute politique face à des adversaires, face aux sophistes à l'origine. Dès lors, ce qui est en jeux dans la thèse idéologique d'Althusser, selon Rancière, c'est le statut des «porteurs du savoir». C'est contre les «faux porteurs du savoir», sophistes, théologiciens, etc., que se lève la tradition épistémologique-métaphysique. Et de même avec Althusser. Le paradigme de cette tradition, selon Rancière, est le cogito cartésien, à partir de l'idée que «la mise en question de l'objet du savoir vise à s'assurer de son sujet. Le doute sur l'objet n'est que l'autre face de la certitude du sujet» (RANCIÈRE, 2011, 242).

Il y a donc une évaluation du projet moderne ici qui doit être prise en compte. Le paradigme cartésien ne servirait qu'à renforcer une domination, un modèle d'explication de la réalité politique dans lequel la distinction savoir versus ignorance semble continuer à opérer. En tant qu'objet d'un litige théorique, même si l'on remarque les différences des auteurs par rapport au modèle de subjectivité cartésien, nous nous demandons dans quelle mesure la modernité ne sert précisément à perpétuer son propre projet, soit par une réponse d'une «lutte croissante pour la reconnaissance», «réformiste», ou par la possibilité d'affirmation de la «part des sans-part», «interventionniste». Si l'on pense à l'exigence morale que Honneth adresse à Althusser, lorsqu'il propose qu'au fur et à mesure que le temps passe plus de critères sont admis pour distinguer des formes idéologiques et des formes justifiées de reconnaissance, ne serait-ce voir dans la lutte pour la reconnaissance un renforcement du même projet progressiste moderne? De même Rancière, en prenant distance avec Althusser et en montrant comment nous sommes encore coincés dans un argument du temps qui nous demande toujours «que nous ne lui manquions pas pour que la politique atteigne sa bonne fin» (RANCIÈRE, 1998, 56-57), n'attire pas précisément notre attention sur la façon dont nous sommes encore modernes? Même si nous voyons dépassé l'âge des naïvetés du progressisme et de la promesse et que l'utopie comme notion spatiale semble vouée à la déception, il n'y aurait plus ce "lieu", I'utopie reste encore comme une notion de temps. Nous rêvons encore d'un temps, un autre temps. En ce sens, et peut-être seulement en ce sens, le rêve de la modernité comme temps à accomplir, comme temps à faire, demeure.

\section{Rancière-Honneth: la proximité et l'éloignement entre les deux critiques}


Rancière arrive alors à ses conclusions concernant l'idéologie. Il nous dit, à la fin de son article, que «le terrain de l'idéologie n'est pas le terrain de l'illusion du sujet en général, des représentations nécessairement inadéquates que les hommes se font de leur pratique» (RANCIÈRE, 2011, 250). Comme Honneth, le philosophe français se pose des questions sur l'idéologie de cette totalité qui enferme. Nous pourrions peut-être établir un parallèle entre les solutions proposées par les deux philosophes. Si Honneth parle du besoin de matérialité pour la reconnaissance d'un sujet, Rancière dit que l'idéologie «n'existe pas simplement dans des discours, pas simplement non plus dans des systèmes, d'images, de signaux, etc. [Elle] existe principalement dans des institutions, dans ce que nous pouvons appeler des appareils idéologiques, au sens où la théorie marxiste parle de I'appareil d'État» (RANCIÈRE, 2011, 250). Il y a donc une matérialité qui aide à comprendre le rôle idéologique; mais, à la différence de Honneth, Rancière semble ne pas penser que cette matérialité puisse, par elle même, fournir aux individus une sorte quelconque d'ouverture face à une reconnaissance idéologique. Une reconnaissance justifiée, différente d'une reconnaissance idéologique, ne semble pas faire partie des projets du philosophe français, d'autant que partant d'une tradition marxiste, critique, soucieuse surtout de «subjectivations politiques», il questionnera ce rôle de la place «légitime», correcte d'un sujet. Partant d'un point de vue politique - et non moral - Rancière questionnera, par exemple, l'identification de termes comme «peuple», «prolétaires», «femmes», avec leurs représentants incarnés. Ces sont des termes litigieux, qui expriment un tort dans leur processus d'identification de reconnaissance, comme il le montre bien dans La Mésentente (1995).

La différence par rapport à Honneth devient peut-être encore plus claire lorsque Honneth affirme que I'«on ne peut rien entendre à ce problème [de la poursuite et de la fin de la lutte des classes dans le domaine de l'idéologie] - et en conséquence ne produire aucune analyse concrète si l'on pose l'idéologie dans le statut de l'illusion» (HONNETH, 2008, 251). C'est sous forme d'illusion que Honneth pensera l'idéologie et proposera à la fin une reconnaissance non-idéologique, «justifiée». Dès lors, nous pourrions dire que Honneth suit le schéma d'Althusser, même si la «science» qui s'oppose à l'idéologie n'est pas la science telle que le philosophe de I'ENS la pense, sur les traces de la tradition épistémologique; mais une «science individuelle», fondée sur la connaissance du sujet face aux contraintes extérieures, dans un apprentissage social universel; c'està-dire, une science que nous pourrions caractériser comme «morale».

En revanche, Rancière ne mettra pas l'idéologie du côté de l'illusion, du «mauvais» côté de I'histoire, comme tout dualisme. Il essaiera de la 
penser en termes de contradiction, dans son sens marxiste, qui est, selon lui, ce qui manque à l'analyse d'Althusser. Comme nous l'avons montré plus loin, en présentant la thèse de Rancière sur l'opposition d'une idéologie à l'autre (I'idéologie dominante opposée à celles des prolétaires), cette stratégie n'a de sens que dans la mesure où nous comprenons que pour Rancière «pour l'entendre [l'idéologie], il faut penser les idéologies comme des systèmes de représentation des intérêts de classe et d'exercice de la lutte des classes» (RANCIÈRE, 2011, 251). Idéologie en lettres italiques et au pluriel, ici, n'est pas un hasard. Dans tout déplacement opéré par Althusser, il semble y avoir une signalisation de concept d'idéologie. D'un système de représentations pluriel, il va, depuis en plus, vers une «universalisation» comme un concept-clé, comme c'est le cas pour «science». Rien de plus métaphysique, donc. Ce que semble intéresser Rancière, cependant, c'est le maintien de ces termes au pluriel; afin de pouvoir travailler avec eux d'une façon incarnée, à l'intérieur de la lutte de classes, engagée non seulement dans des discours et des concepts, mais, surtout, dans des appropriations, acquisitions et maintiens que les idéologies et les sciences «gèrent» au niveau politique.

A partir d'une réflexion sur la révolution culturelle chinoise contemporaine à l'époque - Rancière montre comment la lutte révolutionnaire passe par une confrontation entre «diverses formes de l'idéologie bourgeoise qui se perpétuent après la prise du pouvoir politique: idéologies traditionnelles de l'individualisme ou de la soumission, ou idéologies "modernes" des compétences et de la technicité» (RANCIÈRE, 2011, 252). Même si I'on se méfie du ton de ces termes aujourd'hui, l'important ici est de remarquer le maintien du pluriel du terme «idéologie». Et ce parce que Rancière reste fidèle à l'attitude de penser cette question sous l'angle des effets idéologiques de la division de la société en classes. C'est pour cela qu'il affirme que cette problématique n'a «rien à voir avec la question de la disparition de l'illusion subjective» (RANCIÈRE, 2011, 252).

\section{Conclusion}

Dans une position différente donc de celle de Honneth, Rancière cherche à penser les problèmes de la théorie de l'idéologie d'Althusser à partir de ces conséquences politiques. C'est pour cette raison que la grande critique qui apparaît à la fin de son article - et dans tout le processus de son éloignement d'Althusser qui a lieu à partir du post-Mai 68 - se fera sur le plan du questionnement de l'opposition idéologie/science, ignorance/savoir, comme moteur d'une pensée capable de penser la politique et l'émancipation. Aujourd'hui, cinquante années après cet 
éloignement, dans un monde dominé par des «spécialistes», les paroles de Rancière en 1969 nous semblent encore très actuelles: "La science" devient le mot d'ordre de la contre-révolution idéologique» (RANCIÈRE, 2011 , 254). C'est une certaine interprétation du marxisme qui a permis son propre antidote, lorsqu'elle adopte «la science» d'un statut privilégié pour faire de la politique, entendue, bien évidemment, d'une façon «nonmarxiste». La question ici, bien entendu, n'est pas l'éloge simple de la nonscience, de l'ignorance et du faux. C'est justement dans cet espace que la tradition métaphysique a toujours voulu cantonner ses opposants. La question est donc la reconfiguration de ce schéma dual, entre le vrai et le faux, le savoir et le non-savoir; notamment si I'on veut penser la politique et le statut de la démocratie.

Une «simple» revendication d'un espace normatif à la marge du processus historique et politique des idéologies (comme Honneth semble le soutenir), fondée uniquement sur l'apprentissage et sur le jugement moral du sujet à sa place dans une société donnée, peut engendrer une sorte de dialogue de sourds. $\mathrm{Si}$, pour un individu donné, la matérialité du «travailleur-entrepreneur» ne se réalise pas, rien ne garantit qu'un autre, plus sensible aux discours idéologique du présent puisse y voir cette matérialité. Althusser pourrait, à tout moment, voir de l'idéologie là où Honneth voit de la reconnaissance justifiée. Fonder le critère de reconnaissance sur le sujet, ou - (ce qui semple plus être le cas de Honneth) - sur une tradition rationaliste qui pense en en termes de critères rationnels universaux, peut toujours donner raison à la critique d'Althusser, ainsi qu'à la critique de tout un courant marxiste, y compris Rancière. Il y aura toujours deux niveaux d'analyse; d'où le dialogue de sourds.

Si Rancière ne parle pas de reconnaissance, dans le sens de Honneth, il ne semble pas nonplus se cantonner dans une analyse déconsidérant les sujets, comme cela peut sembler être le cas dans ce texte de 1969. Lorsqu'il cible lutte de classe et essaie de resituer l'idéologie dans cette structure, il ne semble pas s'attendre à trouver dans cette structure une solution pour penser le sujet. À la différence peut-être d'autres marxistes et de toute une tradition «d'espoir» et «de progrès» du marxisme, Rancière ne semble pas doter le sujet d'une reconnaissance du type «vrai» ou, pour employer les termes de Honneth, «justifié». Il n'y a pas de dualisme chez Rancière, «justifié» versus «idéologique», «idéologique» versus «vrai». Si nous pouvons entrevoir un certain type de «reconnaissance» dans la pensée de Rancière, cette reconnaissance sera toujours le résultat d'un processus litigieux d'identification ou de désidentification. Si nous citons l'exemple de Honneth pour la maîtresse de maison, «femme» est pour Rancière 
I'expérience d'un litige, comme il le dit dans La Mésentente (RANCIÈRE, 1995, $60)$.

C'est cela le sens de la femme qui revendique le droit de voter. Elle est reconnue et non reconnue. Elle est reconnue comme «femme» parce qu'elle ne vote pas, parce qu'elle ne doit pas voter, mais aussi parce qu'elle doit le faire. Il ne s'agit pas d'une simple question d'attribution temporelle (comme le disait Honneth) pour obtenir une reconnaissance justifiée ou non, pour elles-mêmes. Le terme même de «femme» est conflictuel, du moins si nous le pensons aussi en termes politiques. Un bon exemple est peut-être le reconnaissance ou nom des femmes dans les propos féministes. Sont-ce des «femmes» parce qu'elles sont d'accord avec ce que pense une théorie féministe donnée, ou sont-ce des «femmes» par qu'elles ne sont pas d'accord? Pour Rancière ce sont des «femmes» dans la mesure où elles peuvent se déplacer entre, qui peuvent entrer et sortir des termes qui les contraignent à se situer dans une place donnée, à une tâche donnée, à ce que I'on appelle «police». Pour revenir au titre de ce travail, si une reconnaissance non-idéologique était possible, il faudrait la penser non seulement en termes de justification, d'un apprentissage rationnel avec d'autres, avec ses expériences, de place que nous devons occuper comme des êtres de nécessités, de droits semblables et d'estime sociale. Ce serait encore être prisonnier d'une sphère policière, car qui assure quels sont les semblables qui ont les mêmes droits que moi, par exemple? «Femmes», sont-ce des semblables? «Prolétaires»? «Gays»? Dans ce sens, pour penser à une reconnaissance non-idéologique, non-policière, il faudrait peut-être penser aux conditions de possibilité de subjectivations politiques, qui nous garantissent, sous forme de litige, de pouvoir occuper momentanément certains espaces de reconnaissance, certains espaces de partage du sensible, comme le dira Rancière. La police finira toujours par revenir, à la dernière minute, lorsqu'une voix est entendue comme étant égale, où elle est reconnue. C'est ce qui s'est passé avec le mouvement ouvrier, avec le mouvement féministe, avec le mouvement pour la diversité. Peu importe, à ce moment-là, et seulement à ce moment-là, peut-être, pourrons-nous parler d'une reconnaissance qui ne prendrait pas la forme d'une idéologie. Une reconnaissance politique qui, en tant que telle, litigieuse, contradictoire, faite d'intervalles. Des intervalles qui persistent, cependant, qui peuvent être rouverts par les mêmes noms: «prolétaire», «femme», «gay». L'espace de la politique - et de la reconnaissance des sujets qui y font partie - est toujours ouvert, toujours prêt à être occupé de façon litigieuse, de façon des-identificatrice. Dans le monde de dissensus de Rancière, «le rapport à soi» est, donc, toujours, d'une certaine façon «désubstantialisé». 


\section{Notas}

1 Professor Formador do Curso de Especialização em Ensino de Filosofia da Universidade Federal de Pelotas (UAB - UFPel).

${ }^{2}$ Voir aussi GENEL, K.; DERANTY, J-P., 2016.

${ }^{3}$ Honneth nous dit qu'il y a de nombreux exemples dans le passé qui pourraient corroborer la thèse d'Althusser, car il y a plusieurs cas où «la reconnaissance manifestée publiquement a en réalité souvent servi uniquement à la constitution et au maintien d'un rapport à soi individuel parfaitement inséré dans le système de la division du travail dominante» (HONNETH, 2008, p. 248). L'éloge de la «bonne» mère de famille et maitresse de Maison proposé par l'Église, les parlements ou les moyens de communication de masse a donné aux femmes, par exemple, une image de soi qui, en réalité, proposait un meilleur format à la division sexuelle du travail.

\section{Références bibliographiques}

ALTHUSSER, L. Réponse à John Lewis. Paris: François Maspero, 1973.

ALTHUSSER, L. Idéologie et appareil Idéologique d'État - Notes pour une recherche, La Pensée, nº 151, juin 1970.

CAMPAILLA, G. Jacques Rancière: dalla rottura com Althusser alle scene dell'emancipazione. Golena edizioni - Contro Cultura, (versão Kindle), 2014.

GENEL, K.; DERANTY, J-P. (eds.) Recognition or Disagreement: a critical encounter on the Politics of Freedom, Equality and Identity. New York: Columbia University Press, 2016.

HONNETH, A. Intégrité et mépris: principes d'une morale de la reconnaissance, Recherches sociologiques n³0, 1999.

HONNETH, A. La lutte pour la reconnaissance, Paris: Gallimard, Folioessais. 2013.

HONNETH, A. La société du mépris, Paris: Éditions de la Découverte, 2008.

HONNETH, A. La Théorie de la reconnaissance : une esquisse, Revue du MAUSS n²3, 2004. 
HONNETH, A. Visibilité et invisibilité: sur l'épistémologie de la "reconnaissance", Revue du MAUSS n² 23, 2004.

MANNHEIM, K. Idéologie et utopie - Une introduction à la sociologie de la connaissance. Paris : Librairie Marcel Rivière et Cie, 1956.

MARX, K.; ENGELS, F. L'idéologie allemande. Paris: Ed. Sociales coll. Classiques du Marxisme, 1974.

RANCIÈRE, J. Aux bords du politique, Paris: La Fabrique éditions, 1998.

RANCIÈRE, J. La Leçon d'Althusser, Paris: La Fabrique éditions, 2011.

RANCIÈRE, J. La Mésentente, Paris: Galilée, 1995.

RANCIÈRE, J. La méthode de l'égalité: Entretien avec Laurent Jeanpierre et Dork Zabunyan. Paris: Bayard, 2012.

RANCIÈRE, J. La nuit des prolétaires: Archives du rêve ouvrier. Paris: Fayard, 1981. 